\title{
Jeffery-Hamel slip flow in a convergent microchannel with uniform wall temperature and streamwise heat conduction
}

\author{
Mohammed NIAGUI*, Youssef HADDOUT, Abdelaziz OUBARRA, Jawad LAHJOMRI* \\ Laboratory of Mechanics, Faculty of Science Ain Chock, Hassan II University, B.P. 5366, Maarif, Casablanca 20100 \\ Morocco \\ *niagui.mohammed@gmail.com* lahjomri@hotmail.com
}

\begin{abstract}
This work is devoted to the determination of the analytical solution of the problem of the laminar forced convection of the Jeffery-Hamel slip flow through a convergent microchannel. The analytical solution is obtained by using a self-adjoint formalism of the functional analysis. The solution represents an extension of the solution obtained in the conventional continuum flow by considering the boundaries slip conditions at the wall and the streamwise heat conduction. This extension has been done by using a new matrix operator of three dimensions in the Hilbert space. The results show that the thermal characteristics are strongly influenced by the Reynolds, Prandtl and Knudsen numbers, the aperture angle of the channel and the streamwise heat conduction.
\end{abstract}

Keywords: Convergent microchannel; Jeffery-Hamel slip flow; self-adjoint formalism; streamwise conduction.

\section{Introduction}

The study of laminar forced convection of the Jeffery-Hamel slip flow through a convergent microchannel is of fundamental and practical importance. This type of flow is often applied in industrial processes using forced convection heat transfer, such as cooling of microelectronic components, micro-heat exchanger design and chemical engineering. This flow is also used in the technology of making plastic sheets and metal sheets through the walls of the microchannel which are heated in part, over a finite length, with a uniform temperature. In the case of a conventional continuum flow regime, from the hydrodynamic point of view, this flow is well known and was first studied by Jeffery in 1915, and then by Hamel in 1917 and then by other authors like Rosenhead [1], Millsaps and Pohlhausen [2]. From the thermal point of view, previous studies of this problem have been carried out as part of boundary layer approximations [3] neglecting radial conduction in the direction of flow. Other works assume that the temperature field develops fully at the entrance of the convergent channel. For example, Riley [4] obtained an analytical solution considering that the temperature field has the same shape as that of the velocity field. Noting that, the phenomena of heat transfer in microscale are completely different compared to those at the macroscale level. The fluid particles that are adjacent to the solid surface do not reach the velocity and the temperature of the wall and have a tangential slip velocity and a temperature different from that of the solid wall thus creating a temperature jump. This slip velocity and temperature jump are related to the Knudsen $K n$ number, which plays an important role at a microscale due to the interaction between molecules and the wall.

This work presents an analytical solution to the problem of forced convection and the development of the temperature field in the thermal entrance region of the Jeffrey-Hamel convergent microchannel in a rarefied environment. This approach is based on a powerful method of self-adjoint formalism. This method results from a decomposition of the energy equation into a system of firstorder partial differential equations. The solution represents an extension to those existing in the earlier works of the continuum flow regime literature; taking into account the slip velocity, the temperature jumps at the wall and the radial conduction. This extension has been obtained by using a new matrix operator of tree dimensions and a scalar product in the Hilbert space. We have shown that this radial conduction has an important role on micro-scale level. The effects of different dimensionless parameters used in this problem, such as Reynolds, Prandtl and Knudsen numbers and the channel aperture angle, on hydrodynamic and thermal characteristics are quantified and analyzed in the slip flow interval range $10^{-3} \leq K n \leq 10^{-1}$.

\section{Mathematical Formulation:}

\subsection{Description of the problem:}

We consider the steady unidirectional, slip flow of a Newtonian fluid entering between two semi-infinite inclined planes separated by an aperture angle $2 \psi$. Figure 1 shows the geometric configuration and the system of the cylindrical coordinates used. The fluid enters with a uniform temperature $\mathrm{T}_{0}$ coming very far from the point formed by these two planes. The walls of the channel are heated with a uniform temperature $\mathrm{T}_{1}$ for $0 \leq \mathrm{r}<r 1$ and taken to the temperature $T_{0}$ for $r \geq r 1$, where $r 1$ is the heating section length. The boundary conditions corresponding to the slip velocity and the temperature jump at the wall are given respectively by Maxwell's and Smoluchowski's formulas:

$$
\begin{aligned}
& u_{r, s}=-\beta_{v} \frac{\lambda}{r} \partial u_{r} /\left.\partial \theta\right|_{\theta=\psi} \\
& T_{s}=T_{0}-\beta_{t} \frac{\lambda}{r} \partial T /\left.\partial \theta\right|_{\theta=\psi}, r \geq r 1, \theta=\psi \\
& T_{s}=T_{1}-\beta_{t} \frac{\lambda}{r} \partial T /\left.\partial \theta\right|_{\theta=\psi}, 0 \leq r<r 1, \theta=\psi
\end{aligned}
$$


$u_{r, s}$ and $T_{s}$ denote the slip velocity and the temperature jump of the fluid at the wall respectively. $\lambda$ is the mean free path of the molecules. $\beta_{v}=(2-F v) / F v$ and $\beta_{t}=$ $(2-F t) / F t 2 \gamma /(\gamma+1) 1 / \operatorname{Pr} . F_{v}$ and $F_{t}$ are the tangential momentum coefficient and thermal accommodation coefficient respectively. Pr and $\gamma$ are Prandtl number and ratio of specific heats respectively.

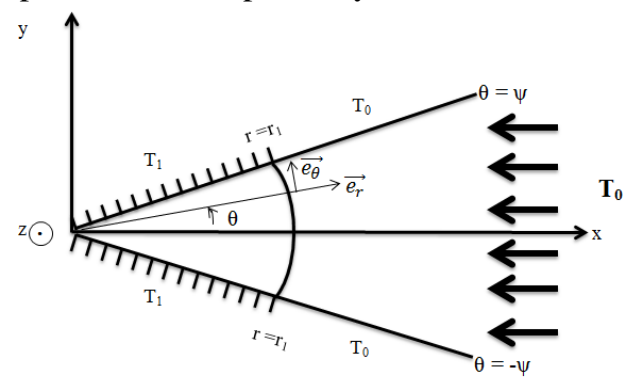

Figure 1 : Geometrical configuration

For an incompressible flow assuming the physical proprieties as constant, neglecting the viscous dissipation and taking into consideration the flow symmetry in term to the $\mathrm{x}$ axis, the dimensionless energy equation considering the streamwise heat conduction in the flow direction can be written:

$U(\eta) \frac{\partial \Theta}{\partial \xi}=\frac{\psi}{P e} \frac{\partial}{\partial \xi}\left[(1-\xi) \frac{\partial \Theta}{\partial \xi}\right]+\frac{1}{\psi P e(1-\xi)} \frac{\partial^{2} \Theta}{\partial \eta^{2}}$

With the dimensionless boundary conditions:

$\frac{\partial \Theta}{\partial \eta}=0$ for $\eta=0,-\infty<\xi<1$

$\Theta \rightarrow 0$, for $\xi \rightarrow-\infty$

$\Theta \rightarrow 1$, for $\xi \rightarrow 1^{-}$

$\Theta(\xi, 1)=\left\{\begin{array}{c}1-\left.C \frac{\partial \Theta}{\partial \eta}\right|_{\eta=1} \text { for } 0<\xi<1 \\ -\left.C \frac{\partial \Theta}{\partial \eta}\right|_{\eta=1} \text { for } \xi \leq 0\end{array}\right.$

Where the dimensionless quantities are defined by:

$\eta=\frac{\theta}{\psi}, \xi=1-\frac{r}{r 1}, U(\eta)=\frac{u_{r}(r, \theta)}{u_{r}(r, 0)}=\frac{F(\theta)}{|F(0)|}$

Where $u_{r}(r, \theta)=\frac{F(\theta)}{r}$ is the velocity field of Jeffery-Hamel flow, $P e=P r . R e=u_{r}(r, 0) r \psi / \alpha, R e=u_{r}(r, 0) r \psi / v=$ $|F(0)| \psi / v$ and $\operatorname{Pr}=v / \alpha$ are respectively the Péclet, Reynolds and Prandtl numbers. Where $F(0)=$ $Q / 2 \psi \int_{0}^{1} U(\eta) d \eta$ is the maximum velocity at the center which is related to the flow rate per unit of length of the channel defined as $Q=\int_{-\psi}^{\psi} r u_{r} d \theta$. The velocity field satisfying the continuity and the Navier-Stokes equations must be solution to the following nonlinear third order differential equation and slip boundary conditions:

$U^{\prime \prime \prime}(\eta)-2 \psi \operatorname{Re} U(\eta) U^{\prime}(\eta)+4 \psi^{2} U^{\prime}(\eta)=0$

$U(0)=1, U^{\prime}(0)=0, U(1)=-\beta_{v} K n U^{\prime}(1)$

where $K n=\lambda / 4 r \psi$ is the Knudsen number and the dimensionless parameter $C$ in Eq. (8) is defined by: $C=$ $4 \beta_{t} K n$.

\subsection{Analytical solution:}

By defining the dimensionless axial energy flow [5]:

$\varphi(\xi, \eta)=\int_{0}^{\eta}\left[U\left(\eta^{\prime}\right) \Theta\left(\xi, \eta^{\prime}\right)-\frac{\psi}{P e}(1-\xi) \frac{\partial \Theta}{\partial \xi}\right] d \eta^{\prime}$

allows to decompose the energy equation into a system of two a first order partial differential equation:

$P e \psi(1-\xi) \frac{\partial \Theta}{\partial \xi}=P e^{2} U(\eta) \Theta-P e^{2} \frac{\partial \varphi}{\partial \eta}$

$P e \psi(1-\xi) \frac{\partial \varphi}{\partial \xi}=\frac{\partial \Theta}{\partial \eta}$

By applying a self-adjoint formalism. [5], the solution for the dimensionless temperature $\Theta(\xi, \eta)$ can be represented as an infinite series of eigenfunctions $\phi_{n 1}^{+}(\eta)$ and $\phi_{n 1}^{-}(\eta)$ (i.e. the first components of vectors $\vec{\phi}_{n}^{ \pm}$):

$\Theta(\xi, \eta)=-\sum_{n=1}^{\infty} A_{n}(1-\xi)^{-\frac{\lambda_{n}^{2}}{P e \psi}} \phi_{n 1}^{+}(\eta)$ for $\xi \leq 0$

$\Theta(\xi, \eta)=1-\sum_{n=1}^{\infty} B_{n}(1-\xi)^{\frac{\beta_{n}^{2}}{P e \psi}} \phi_{n 1}^{-}(\eta)$ for $0<\xi<1(16)$

With $\mu_{n}^{+}=\lambda_{n}^{2}$ and $\mu_{n}^{-}=-\beta_{n}^{2}$, where the expansion coefficients $A_{n}$ and $B_{n}$ are given explicitly as:

$A_{n}=\frac{2}{\lambda_{n}\left[\frac{d \phi_{n 1}^{+}(1)}{d \lambda_{n}}+C \frac{d \phi_{n 1}^{+1}(1)}{d \lambda_{n}}\right]}$ and $B_{n}=-\frac{2}{\beta_{n}\left[\frac{d \phi_{n 1}^{-}(1)}{d \beta_{n}}+C \frac{d \phi_{n 1}^{-1}}{d \beta_{n}}\right]}$

\section{Results and discussion}

The validity of our analytical solution could be checked, in the simplified case of continuum flow $(K n=0)$ and in the absence of streamwise heat conduction, by comparing the first five eigenvalues $\beta_{n}$ and the corresponding expansion coefficients $B_{n}$ for a convergent channel, with the solution of Vachagina et Ananyev [4]. This comparison is illustrated in Table 1 for $\psi=0.57^{\circ}$ $P r=7$ et $R e=1000$. It is clear, that the agreement between both calculations is excellent.

Table 1: The first five eigenvalues $\beta_{n}$ and the corresponding expansion coefficients $B_{n}$ for a convergent channel obtained from analytical solution, with the results of Vachagina and Ananyev (IJHMT, 2013) [6], in the absence of streamwise heat conduction and rarefaction.

\begin{tabular}{|c|c|c|c|c|c|}
\hline $\mathrm{n}$ & $\beta_{n}^{2}[6]$ & & $B_{n}[6]$ & $\beta_{n}^{2}$ This work & $B_{n}$ This work \\
\hline 1 & 2.62081 & & 1.22556 & 2.620816245 & 1.22556567 \\
\hline 2 & 26.9795 & & -0.336629 & 26.97955929 & -0.3366292679 \\
\hline 3 & 78.0081 & & 0.181712 & 78.00803684 & 0.1817122862 \\
\hline 4 & 155.686 & & -0.121458 & 155.6860835 & -0.1214583047 \\
\hline 5 & 260.008 & & 0.0900475 & 260.0064925 & 0.09004750887 \\
\hline
\end{tabular}

Figure 2 represents the influence of the channel angle and Knudsen number on the bulk temperature for convergent microchannel. For large value of angle $\left(\psi=20^{\circ}\right)$ and for a given $K n$, the uniform temperature profile for each curve is established for a negative axial distance in the upstream section very far from the entrance, indicating that the temperature field in the microchannel is very affected by the effect of streamwise heat conduction. The reason is that the 
heat generated from the wall which is progressively transmitted to the fluid in the downstream heating section, a part of this heat is transferred towards the upstream of the entrance section $\xi=0$. By decreasing the value of angle to $\psi=5^{\circ}$, the effect of streamwise heat conduction is very small and the bulk temperature on the upstream of entrance section decreases due to cooling effect of convection. Therefore, at the entrance section, the temperature starts very closely to $\xi=0$ with a value of approximately zero.

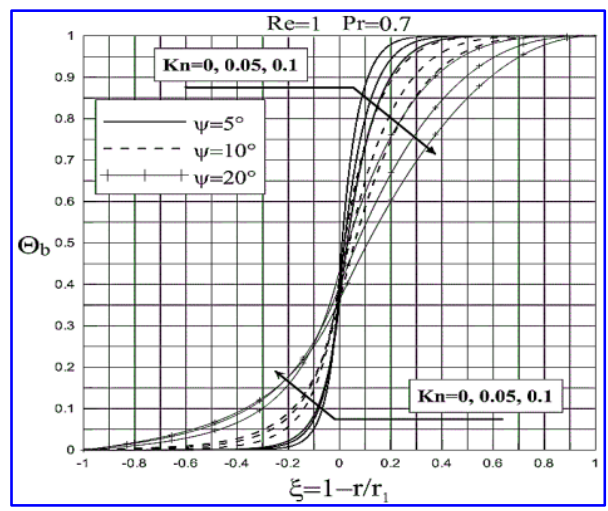

Figure 2: Effect of angle $\psi$ and $K n$ on $\Theta_{b}$ distribution for $R e=1$ and $\operatorname{Pr}=0.7$

Figure 3 shows the variation of the local Nusselt number $N u$ with the radial distance for different values of angle $\psi$ of the channel and Knudsen numbers in a convergent microchannel. It is noted that, for a given value of $K n$ and $\psi, N u$ decreases monotonically with $\xi$ in the thermal entrance region because less heat transfer occurs from the wall to the fluid as the fluid is heated downstream. Farther downstream at large $\xi$, the flow becomes thermally developed and Nusselt number value approaches a fixed value. For a given $K n$, the effect of $\psi$ leads to increase the local Nusselt number. Indeed, as $\psi$ increases, streamwise heat conduction on the flow in convergent microchannel is significant which leads to increase the heat exchange. By inspecting figure 3, as Knudsen number increases, Nusselt number decreases due to the reduction in heat transfer from the wall to the fluid. Increasing Knudsen number leads to increased temperature jump at the wall. As a result, the adjacent fluid to the wall does not feel the real temperature of the wall. This result in lower heat flux from the wall to the fluid and so Nusselt number decreases. The reduction in $\mathrm{Nu}$ due to the increase in temperature jump dominates the increase in $N u$ due to the increase in fluid flow rate and as a net result, $N u$ decreases as $K n$ increases.

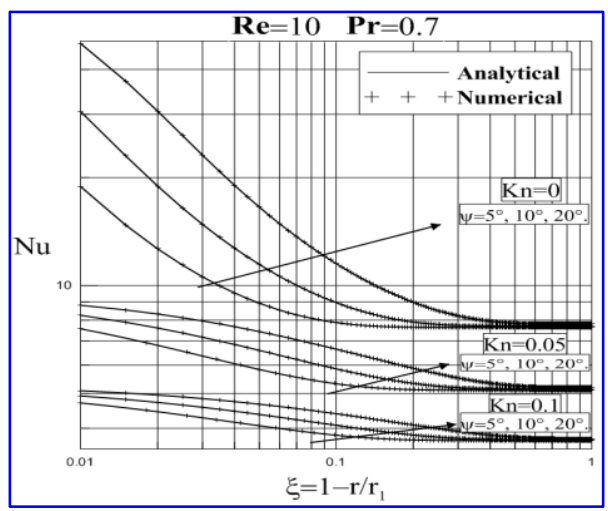

Figure 3: Effect of angle $\psi$ and $\mathrm{Kn}$ on $\mathrm{Nu}$ distribution for $\mathrm{Re}=10$ and $\operatorname{Pr}=0.7$
Notice that Fig. 3 gives also a comparison between the analytical solution and the numerical solution of energy equation (4)-(8), based on the finite difference method. The comparison indicates clearly that the two solutions are in excellent agreement.

\section{Conclusion}

In this paper, the solution of forced convection of a JefferyHamel flow is obtained both analytically and numerically. The analysis includes the streamwise heat conduction and slip boundary conditions. The solution is compared to the simplified limiting case in the absence of streamwise heat conduction available in the literature and a good agreement is found. From the results obtained the following conclusions can be drawn.

1) In the presence of rarefaction, the effect of streamwise heat conduction on the flow in convergent microchannel is significant for small Reynolds number and for large values of aperture angle of the channel.

2) It can be concluded that the heat exchange decreases with increasing the effect of slip characterized by Knudsen number. This decrease is due essentially to the temperature jump at the wall.

3) In the absence and in the presence of the rarefaction, the heat exchange increases with increasing the Reynolds number or increasing of the aperture angle of the channel.

The results obtained in this study can be very helpful and applied directly to the field of cooling technology for microelectronic systems.

\section{References}

[1] L. Rosenhead, The steady two-dimensional radial flow of viscous fluid between two inclined plane walls, Proc. R. Soc. Lond., A 175 (1940), 436-467.

[2] K. Millsaps and K. Pohlhausen, Thermal distributions in Jeffery-Hamel flows between nonparallel plane walls, Journal of the Aeronautical Sciences, Vol. 20, No. 3 (1953), pp. 187-196.

[3] J. Dey, G. Nath, Boundary Layer Convective Heat Transfer in a Convergent Channel, Letters in Heat and Mass Transfer, vol. 8, pp. 371-377, 1981.

[4] N. Riley. Heat Transfer in Jeffery-Hamel Flow, Q. Journal Mech. Appl. Math., vol. 42, pp. 203-211, 1988.

[5] Y. Haddout, J. Lahjomri, The extended Graetz problem for a gaseous slip flow in micropipe and parallel-plate microchannel with heating section of finite length: Effects of axial conduction, viscous dissipation and pressure work, International Journal of Heat and Mass Transfer, vol. 80, pp 673-687, 2015.

[6] E. K. Vachagina, D. V. Ananyev, Fourier method for heat transport equation in the convergent channel, International Journal of Heat and Mass Transfer, vol. 57, pp. 148-154, 2013. 